\title{
Fan-shaped jets above the light bridge of a sunspot driven by reconnection ${ }^{\star}$
}

\author{
Carolina Robustini ${ }^{1}$, Jorrit Leenaarts ${ }^{1}$, Jaime de la Cruz Rodriguez ${ }^{1}$, and Luc Rouppe van der Voort $^{2}$ \\ ${ }^{1}$ Institute for Solar Physics, Department of Astronomy, Stockholm University, AlbaNova University Centre, \\ 10691 Stockholm, Sweden \\ e-mail: carolina.robustini@astro.su.se \\ 2 Institute of Theoretical Astrophysics, University of Oslo, PO Box 1029 Blindern, 0315 Oslo, Norway
}

Received 21 December 2015 / Accepted 1 April 2016

\begin{abstract}
We report on a fan-shaped set of high-speed jets above a strongly magnetized light bridge (LB) of a sunspot observed in the H $\alpha$ line. We study the origin, dynamics, and thermal properties of the jets using high-resolution imaging spectroscopy in $\mathrm{H} \alpha$ from the Swedish 1m Solar Telescope and data from the Solar Dynamics Observatory and Hinode. The $\mathrm{H} \alpha$ jets have lengths of 7-38 Mm, are impulsively accelerated to a speed of $\sim 100 \mathrm{~km} \mathrm{~s}^{-1}$ close to photospheric footpoints in the LB, and exhibit a constant deceleration consistent with solar effective gravity. They are predominantly launched from one edge of the light bridge, and their footpoints appear bright in the $\mathrm{H} \alpha$ wings. Atmospheric Imaging Assembly data indicates elongated brightenings that are nearly co-spatial with the $\mathrm{H} \alpha$ jets. We interpret them as jets of transition region temperatures. The magnetic field in the light bridge has a strength of $0.8-2 \mathrm{kG}$ and it is nearly horizontal. All jet properties are consistent with magnetic reconnection as the driver.
\end{abstract}

Key words. techniques: imaging spectroscopy - Sun: chromosphere - Sun: photosphere - sunspots

\section{Introduction}

Complex sunspots can exhibit light bridges (LBs) crossing the sunspot umbra. The chromosphere above LBs is rich in dynamic phenomena (e.g., Berger \& Berdyugina 2003; Louis et al. 2008, 2009). Jets of cool material $(<15 \mathrm{kK})$ are observed above certain LBs; a detailed investigation of their dynamics is due to Roy (1973a) who extrapolated the full velocity vector of four jets observed in $\mathrm{H} \alpha$, combining the plane-of-the-sky (POS) velocity of the jet fronts and the magnetic field inclination obtained from a potential field extrapolation (Roy 1973b). According to his analysis these jets can reach a maximum speed of $175 \mathrm{~km} \mathrm{~s}^{-1}$ and extend up to $50 \mathrm{Mm}$. They accelerate during an extended amount of time until they reach maximum velocity at a height between 7 and $50 \mathrm{Mm}$. Then they show an ascending phase where they decelerate more quickly than the effective solar gravitational deceleration, and subsequently a descending phase where the acceleration is smaller than the effective gravity. In order to explain this behavior Roy (1973b) suggested a braking force due to perturbation in the potential field that opposes the motion of the jets.

Light bridge jets have been studied more recently by other authors. Asai et al. (2001) have reported on dark recurrent $\mathrm{H} \alpha$ jets with apparent length of up to $23 \mathrm{Mm}$ and visible in the extreme-ultraviolet channels of the Transition Region and Coronal Explorer (TRACE). These jets exhibit a fan-shape also recognizable in the observations of Roy (1973a). Bharti et al. (2007) have found mass ejections in a LB with a line-of-sight magnetic field whose polarity was opposite that of the umbral field. Shimizu et al. (2009) and Louis et al. (2014) observed shorter jets emanating from one edge of a LB with an apparent length of a few Mm using the wide-band $\mathrm{Ca}$ II $\mathrm{H}$ filter on board

\footnotetext{
* Movies associated to Figs. 1 and 2 are available in electronic form at http://www . aanda.org
}

the Hinode spacecraft. Bharti (2015) and Toriumi et al. (2015a) have reported on similar jets showing a parabolic motion in the POS. They both used observations from Hinode, the Interface Region Imaging Spectrograph (IRIS) and the Atmospheric Imaging Assembly (AIA, Lemen et al. 2012) on board the Solar Dynamic Observatory (SDO).

In this paper we report on the dynamics and thermal structure of recurrent jets above a LB with the same fan shape, similar velocity, and apparent length as reported by Roy (1973a) and Asai et al. (2001). We do so using imaging spectroscopy in $\mathrm{H} \alpha$ and imaging using various AIA channels. Unlike Roy (1973a), we give the entire dynamic description independently of the inferred magnetic field vector. Because of their fan shape and beauty in $\mathrm{H} \alpha$, we call them peacock jets. In lower resolution observations they are referred to as surges.

\section{Observations and data reduction}

The jets were observed in AR11785 in its decaying phase on 2013 July 5 between 8:11 and 9:38 UT with the CRisp Imaging SpectroPolarimeter (CRISP, Scharmer et al. 2008) at the Swedish 1m Solar Telescope (SST, Scharmer et al. 2003). Its heliocentric coordinates were $(x, y)=\left(-485^{\prime \prime},-211^{\prime \prime}\right)$ corresponding to an observing angle of $32^{\circ}(\mu=0.85)$. The measurement cadence was $8.8 \mathrm{~s}$ with 39 positions along the line profile between $+95 \mathrm{~km} \mathrm{~s}^{-1}$ and $-95 \mathrm{~km} \mathrm{~s}^{-1}$ Doppler shift relative to line center and a spacing of $5 \mathrm{~km} \mathrm{~s}^{-1}$. The pixel scale was 0 '”058. The CRISP data were reduced following the pipeline described in de la Cruz Rodríguez et al. (2015), which includes image restoration with Multi-Object Multi-Frame Blind Deconvolution (MOMFBD, van Noort et al. 2005). We also use coaligned co-temporal data taken with the AIA and the Helioseismic and Magnetic Imager (HMI, Scherrer et al. 2012) on board 

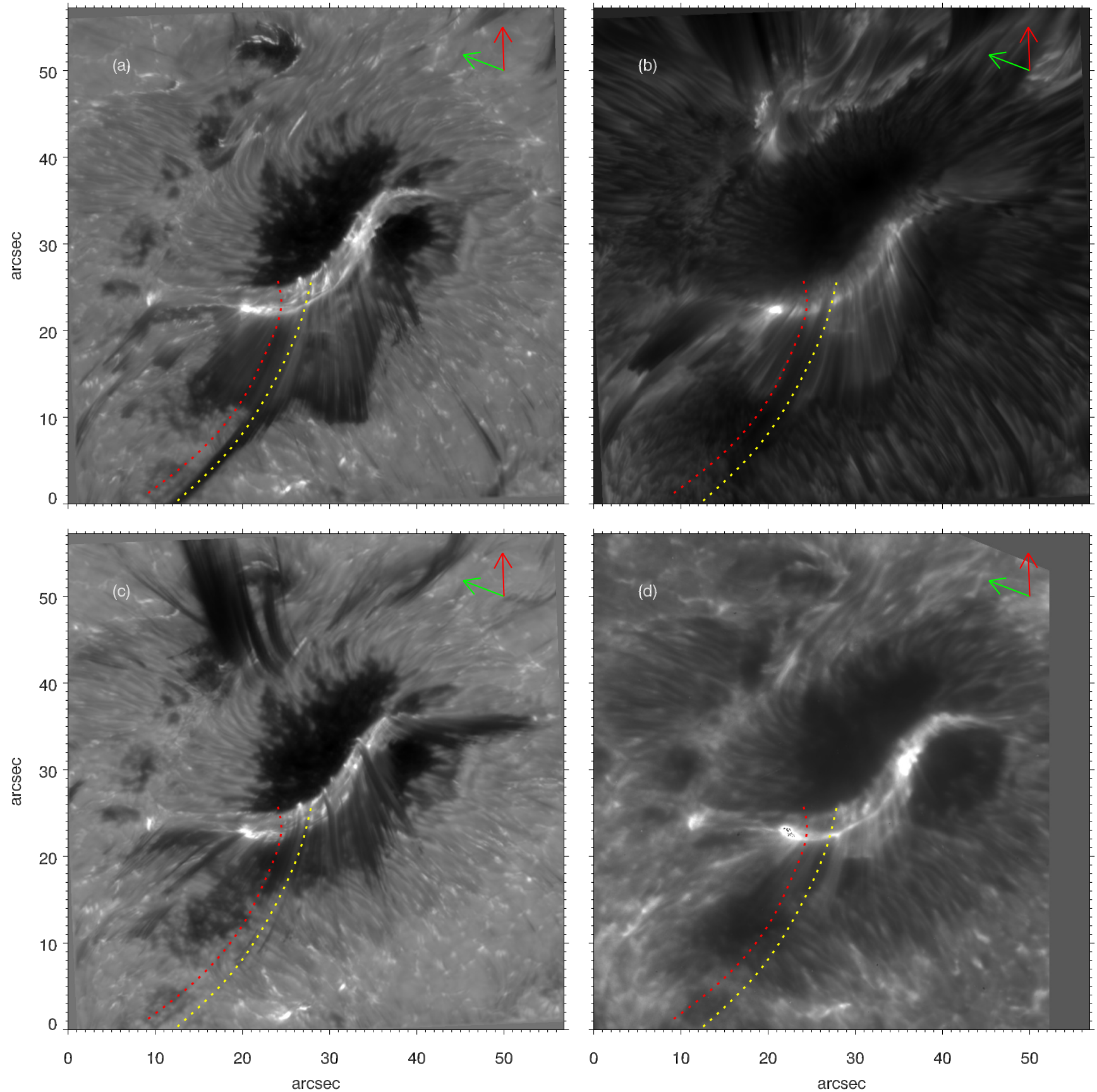

Fig. 1. Peacock jets observed in the $\mathrm{H} \alpha$ line on 2013 June 5 at 9:23 UT. a) Blue wing image at $\Delta \lambda=-45 \mathrm{~km} \mathrm{~s}^{-1}$; b) the line core; c) red wing image at $\Delta \lambda=45 \mathrm{~km} \mathrm{~s}^{-1}$; d) co-temporal wide-band image in the SOT Ca II H filter. The red and yellow dashed curves are the trajectories of the jets shown in Figs. 4 and 5. The red and green arrows point toward the solar disk center and the solar north pole, respectively. This figure is accompanied by an online animation that shows the temporal evolution of panels a)-c).

SDO. The cadence of the AIA and HMI was $12 \mathrm{~s}$ and $48 \mathrm{~s}$, respectively. They have both been resampled to match the cadence and pixel size of the SST data. The alignment was performed by first rotating the relevant SDO subfield to the same orientation as the SST data and then performing a cross correlation between the SST H $\alpha$ wide-band data and the HMI continuum data. The co-alignment is accurate to within an SDO pixel size of 0 ".5. The SDO data show that the jets were present from approximately 2013 July 4, 20:00 UT, to July 5, 22:00 UT.

In addition we use partially overlapping Hinode (Kosugi et al. 2007) observations, consisting of a Solar Optical Telescope (SOT, Tsuneta et al. 2008) wide-band Ca II H image taken at 09:23 UT and a SOT-spectropolarimeter fast raster in Fe 16302 taken between 8:14 and 8:46 UT.

We used the CRisp SPectral EXplorer (CRISPEX, Vissers \& Rouppe van der Voort 2012) software to analyze the data.

\section{Results}

Figure 1 shows the peacock jets in the blue and red wings of $\mathrm{H} \alpha$ and in the line center a-c and $\mathrm{Ca}$ II $\mathrm{H}$ d. The jets appear as slightly curved, dark absorption features in the blue and red wing images. They are less clear in the $\mathrm{H} \alpha$ line core image and in 
Ca II $\mathrm{H}$ where they appear weakly in emission against the background of the superpenumbra. The panels $a, b$, and d show a pattern of shorter and brighter jets launched together with the longer ones. The blueshifted and redshifted absorption features are not co-spatial. The blue-wing features appear predominantly rooted in a chain of finely structured brightenings at the lower edge of the LB (Fig. 1a). In some cases the red-wing features extend to the upper edge of the LB c. The Ca II $\mathrm{H}$ jets have the same appearance as in Shimizu et al. (2009).

Figure 2 compares the appearance of the $\mathrm{H} \alpha$ jets with the magnetic field vector in the local reference system and the appearance in the AIA channels. The field strength $b$ and the local-frame inclination map c are obtained from an azimuth deambiguated inversion with a Milne-Eddington atmosphere done with MERLIN (Skumanich \& Lites 1987). To solve the $180^{\circ}$ ambiguity we applied the acute angle method (Sakurai et al. 1985): we assumed a potential field and imposed that the LOS observed magnetic field and the transverse potential field components make an angle smaller than $90^{\circ}$.

In the LB the field is more horizontal and weaker $(0.8-2 \mathrm{kG})$ than the more vertical umbral field, which has a strength of $2-3.3 \mathrm{kG}$. The horizontal field component in the $\mathrm{LB}$ is aligned to the LB itself. A similar configuration is reported by Toriumi et al. (2015a), but with a more weakly magnetized LB. The simulations of Toriumi et al. (2015b) suggest that the jets observed in Toriumi et al. (2015a) can be driven by magnetic reconnection between the horizontal field carried into the LB and the vertical field of the surrounding umbra. The LB field is weaker than the umbral field (2.2-3.2 kG).

Figure 3 shows the profile of the magnetic field strength along three lines (A, B, and C of Fig. 2b) crossing the length of the LB at right angle.

The bright jet footpoints are located where the polarity of the $B_{z}$ field changes. The footpoints appear bright in the $1700 \AA$ and $1600 \AA$ channels providing further evidence that they are indeed located in the upper photosphere or lower chromosphere (Fossum \& Carlsson 2005). Some of the brightest footpoints (e.g., footpoint marked 1 in $\mathrm{H} \alpha$ and $171 \AA$ ) appear in all the AIA channels. All channels display an elongated brightening coaligned with the direction of the $\mathrm{H} \alpha$ jet. All channels except $94 \AA$ continuously show a bright rim at the location of the top of the $\mathrm{H} \alpha$ jets, and intermittently also in $94 \AA$.

Figures 4 and 5 show space-time diagrams of the intensity in $\mathrm{H} \alpha$ and AIA channels along the trajectories indicated by the dashed lines in Fig. 1. Panels a-c show the evolution in the blue wing, line center, and red wing of $\mathrm{H} \alpha$. The jet fronts appear as parabolic trajectories with the upward part most clearly seen in the blue wing, the tops in the line-center, and the downward part more visible in the red wing. The red curves show a parabolic fit to the front of two jets.

In Fig. 4 the launch of the first jet coincides with a footpoint brightening at $0.7 \mathrm{ks}$, while for the second jet no intensity enhancement is visible on the LB. This occurs because the jets sometimes show sideways motion, and the trajectory misses the location of the brightening, but it is visible in the online animation of Fig. 1. At $2.6 \mathrm{ks}$ a strong brightening is visible on the LB, but no dark jet is associated with it; on the contrary, we observe a shorter parabolic feature slightly in emission against the umbral background (see the animation at 08:50 UT).

The space-time diagram in Fig. 5a exhibits more prolonged brightenings on the LB accompanied by three clear subsequent jets at $0.7 \mathrm{ks}, 2.4 \mathrm{ks}$, and $3.3 \mathrm{ks}$. Most, but not all, jets emanate from brightenings at the lower edge of the light bridge, labeled with the position L1 in Figs. 4 and 5.

Panels d-e show the intensity in three AIA channels. The jet fronts always appear bright in $171 \AA$ and $304 \AA$. The three strongest $\mathrm{H} \alpha$ footpoint brightenings at $0.7 \mathrm{ks}, 2.5 \mathrm{ks}$, and $5.0 \mathrm{ks}$ (Fig. 4) coincide with bright jet-aligned streaks in all AIA channels. The AIA channels in Fig. 5 do not show such dramatic events on the LB except the saturated pixels around $5 \mathrm{ks}$. Panel $\mathrm{f}$ shows the light curves for $\mathrm{H} \alpha$, AIA $171 \AA$, and $304 \AA$. The jetaligned streaks in AIA d-e coincide with a sudden increase in intensity in the blue wing of $\mathrm{H} \alpha$ (black), in AIA $171 \AA$ (blue), and $304 \AA$ (brown). The peaks in $\mathrm{H} \alpha$ exhibit a slower decay than in the AIA channels. The jets appear as intensity drops when the jets move upward (turquoise, blue wing) and downward (orange, red wing) at position L2.

Finally, panels $\mathrm{g}$ and $\mathrm{h}$ show $\lambda-t$ plots of $\mathrm{H} \alpha$ along the lines labeled L1 and L2 in panels a-c. The spectrum at the footpoints shows many impulsive intermittent brightenings in the wings that last from approximately fifty to several hundred seconds. Many but not all brightenings show a corresponding subsequent parabolic jet profile in the space-time diagrams. The stronger brightenings in Fig. 4 coincide with the three intensity bumps in panel $g$ of Fig. 4, which extend all along the $\mathrm{H} \alpha$ profile. On the contrary the bright footpoints of Fig. 5 have intensity bumps as a counterpart, mostly in the blue wing. Some jets associated with the other brightenings follow slightly different trajectories and are thus absent or partially visible. The three strongest brightenings have partially or completely filled in line-cores (see also Fig. 7).

Panel h shows a standard $\mathrm{H} \alpha$ background profile. Strong diagonal absorption features caused by the jets are superimposed with maximum blueshifts and redshifts higher than $\pm 75 \mathrm{~km} \mathrm{~s}^{-1}$. The near linear shift from blueshift to redshift indicates constant deceleration of the jet material.

From Figs. 1-4 and the associated movies the following qualitative picture arises: fast high-reaching jets of plasma with temperatures lower than $15 \mathrm{kK}$, are continuously launched during the full 1.5-h duration of our observing sequence. Their footpoints are located at the lower edge of a light bridge, where the field configuration (see panel c of Fig. 2) suggests a magnetic shear between the LB horizontal field and the umbral field. They appear bright in $\mathrm{H} \alpha$ and AIA $1700 \AA$ and occasionally even in hotter AIA channels, and must therefore be located in the photosphere or low chromosphere. The jets are not launched at random times and locations. Instead, the footpoint brightenings appear to "ripple" along the LB (see the online movies). Once accelerated, the jet material follows a ballistic trajectory and falls back to the solar surface. Some jets fall back to the upper edge of the LB, and Fig. 4 shows evidence of almost co-spatial hot (AIA) and $\operatorname{cool}(\mathrm{H} \alpha)$ jets. The presence of co-spatial cold and hot jets is consistent with simulations of flux emergence into pre-existing vertical field (e.g., Yokoyama \& Shibata 1995).

The jets develop a bright front in the AIA channels that we tentatively interpret as the signature of compressive density increase and heating as the jet rams into the coronal material. However this brightening is also shown by the down-flowing material. This indicates that the physical process behind it is more complex and requires further investigation.

We then proceeded to quantify the jet dynamics. We assumed that the jets move along straight lines. Figure 1 and the associated movie indicate that they do not. However, the deviations from a straight line are not large, so the error we make is likely 

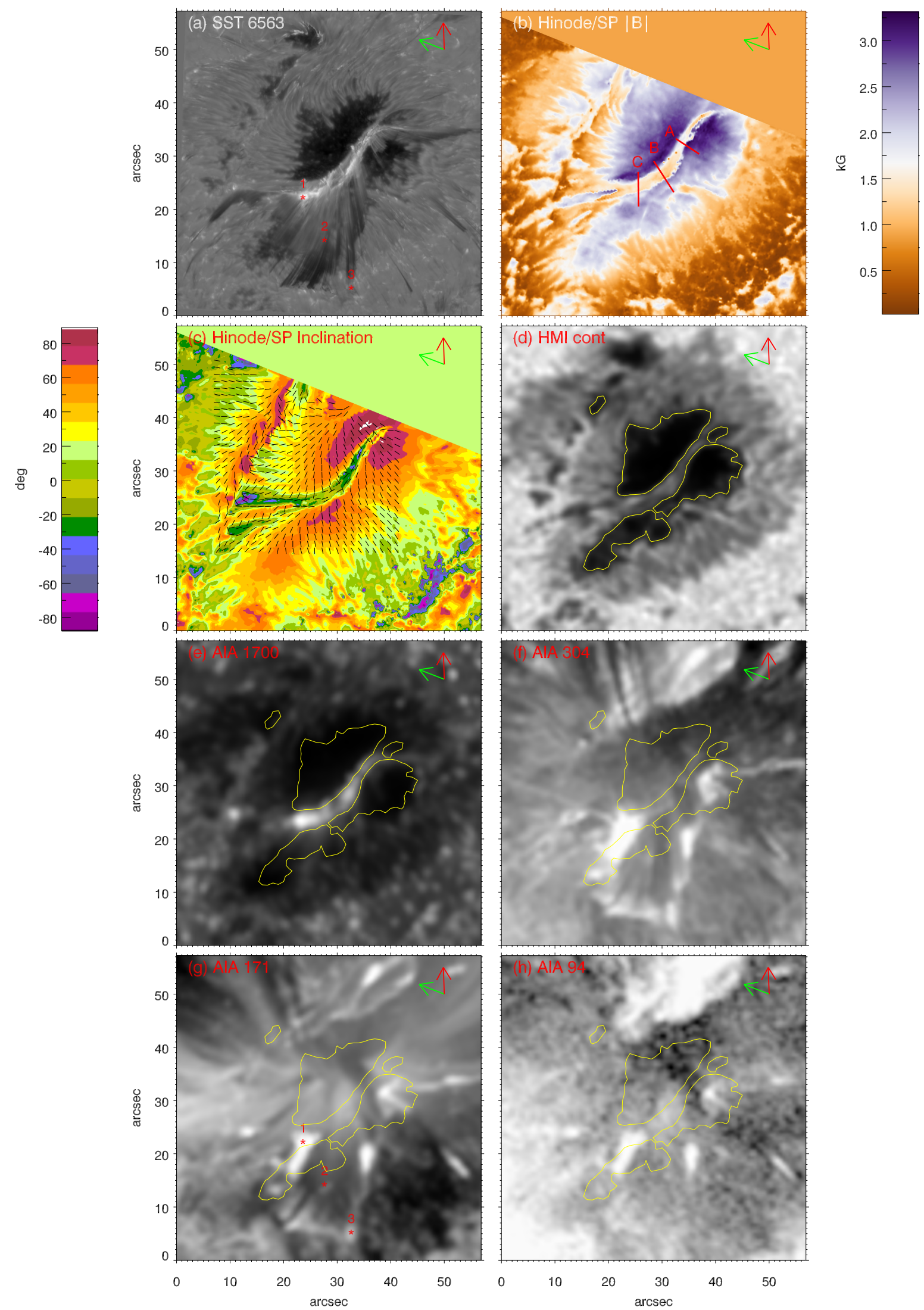

Fig. 2. a) Peacock jets in the blue wing of $\mathrm{H} \alpha$; b) magnetic field strength $|\boldsymbol{B}|$. The lines $\mathrm{A}, \mathrm{B}$, and $\mathrm{C}$ refer to Fig. 3; c) magnetic field inclination with respect to the solar vertical and the direction of the horizontal component of the magnetic field (line segments). Panels d)-h) show images in the different AIA filters co-temporal with the $\mathrm{H} \alpha$ image. The yellow contour outlines the umbra. The numbered stars indicate points whose DEM is shown in panel c) of Fig. 7. This figure is accompanied by an online animation where the single Hinode SP scan b) is replaced by the temporal evolution of the HMI magnetograms and the inclination map c) by AIA 1600. 


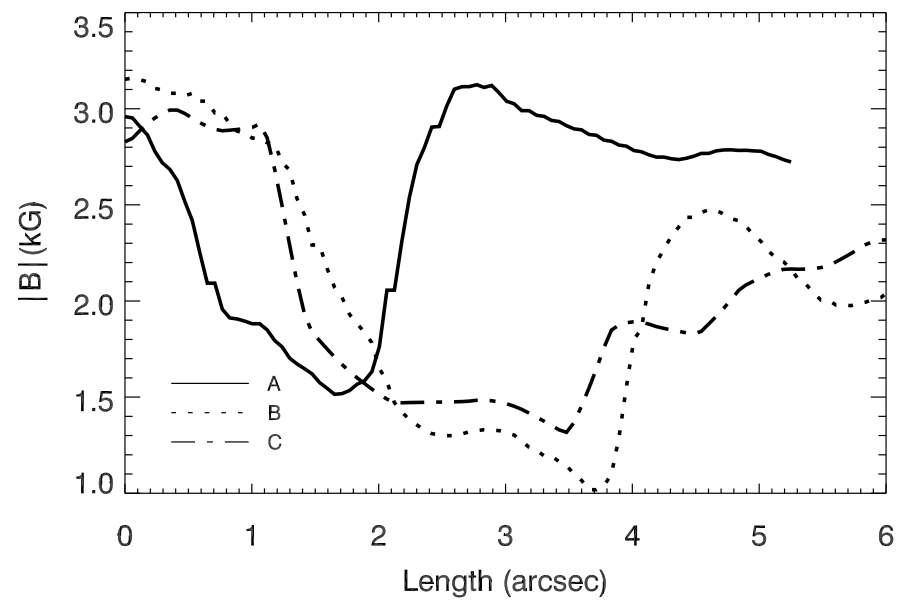

Fig. 3. Magnetic field strength profile plotted along the three lines A, B, and $\mathrm{C}$ in Fig. $2 \mathrm{~b}$.

to be small compared to the accuracy with which we can measure the velocity and deceleration of the jets.

We traced the POS of all jets that show a clearly defined ascending and descending trajectory (40 jets in total) in $\mathrm{H} \alpha$ and extract the $\lambda-s-t$ data along the path. Then, based on $s-t$ cuts we fitted the POS motion of the jet front with a parabola (see examples in panels a-c of Figs. 4 and 5). We then extracted $\lambda-t$ slices for several positions along the parabola and measure the line-of-sight (LOS) velocity of the jet front from the maximum Dopplershift of the absorption feature (as in panel h of Figs. 4 and 5), while we obtained the LOS deceleration from the slope of the absorption feature in the $\lambda-t$ slice. Using these measurements, we fitted a linear function to the velocity as a function of time of the jet front along the LOS. Based on the dynamics of the jet fronts along the LOS and in the POS, we determined their distance from the photospheric footpoint $s$ as a function of time, the initial velocity $v_{0}$, and deceleration $a$,

$$
\begin{aligned}
& s(t)=v_{0}\left(t-t_{0}\right)-\frac{a}{2}\left(t-t_{0}\right)^{2} \\
& v_{0}=\left(v_{\mathrm{LOS}}^{2}\left(t_{0}\right)+v_{\mathrm{POS}}^{2}\left(t_{0}\right)\right)^{1 / 2} \\
& a=\left(a_{\mathrm{LOS}}^{2}+a_{\mathrm{POS}}^{2}\right)^{1 / 2},
\end{aligned}
$$

where $t_{0}$ is the time the fitted parabola along the POS motion crosses the bright edge of the light bridge. The initial velocity is thus an estimate of the jet velocity directly after the impulsive acceleration.

From the position of the jets on the solar disk and the POS and LOS velocities we computed the angle $\theta$ of the jet with the solar vertical assuming motion along a straight line using the procedure outlined in Title et al. (1993), which allows us to compare the deceleration of the jet to the effective gravity $g \cos \theta$, with $g$ the solar gravitational acceleration.

In Fig. 6 we show histograms of the jet angle with the vertical, initial jet velocity, maximum jet length, and $a / \cos \theta$. The last quantity would be equal to the solar gravity if gravity were the only force acting on the jet. The jets make an angle of $25^{\circ}$ with the LOS. Owing to the large thermal width of the $\mathrm{H} \alpha$ line, and the superposition of multiple jets along the LOS we predict typical errors of $10 \mathrm{~km} \mathrm{~s}^{-1}$ in the determination of the Doppler shift of the absorption features in the spectra, and $15 \%$ relative error in the LOS deceleration. The POS velocity and deceleration can be determined with $5 \%$ error. This leads to errors in

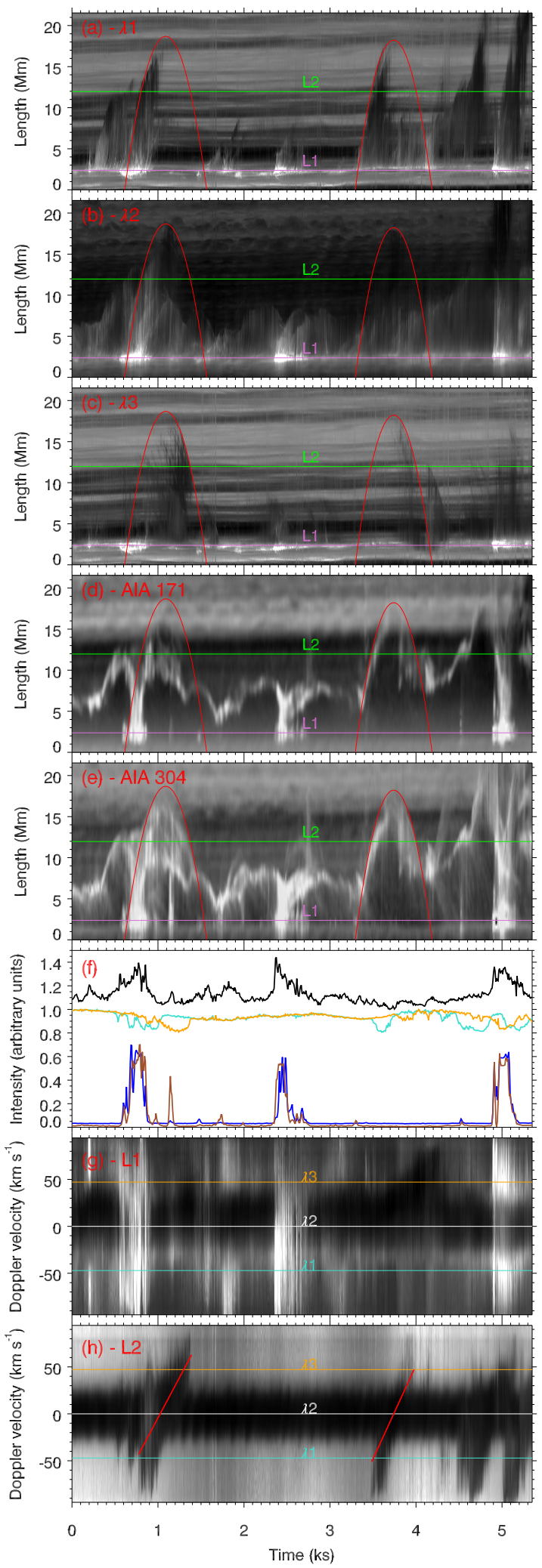

Fig. 4. Panels a)-c): time-slices ( $s$ - $t$ plots) along the red trajectory in Fig. 1 for $\mathrm{H} \alpha-50 \mathrm{~km} \mathrm{~s}^{-1}$ a), $\left.\mathrm{H} \alpha 0 \mathrm{~km} \mathrm{~s}^{-1} \mathbf{b}\right), \mathrm{H} \alpha+50 \mathrm{~km} \mathrm{~s}^{-1} \mathbf{c}$ ), and two AIA channels d)-e). The red parabolas are fits to $\mathrm{H} \alpha$ jet fronts. Panel f): normalized light curves for $\mathrm{H} \alpha$ at $\lambda 1$ at location L2 (turquoise), $\mathrm{H} \alpha$ at $\lambda 3$ at L2 (orange), $\mathrm{H} \alpha$ at $\lambda 1$ at L1 (black), AIA 171 at L1 (blue), and AIA 304 at L1 (brown). Panels g)-h): time evolution of the $\mathrm{H} \alpha$ spectrum along the lines labeled L1 and L2 in panels a)-e). The lines labeled $\lambda 1-\lambda 3$ are the wavelengths for which a time-slice is shown in panels a)-c). The red lines in panel h) are fits to the Doppler shift of the absorbing feature associated with a jet. 

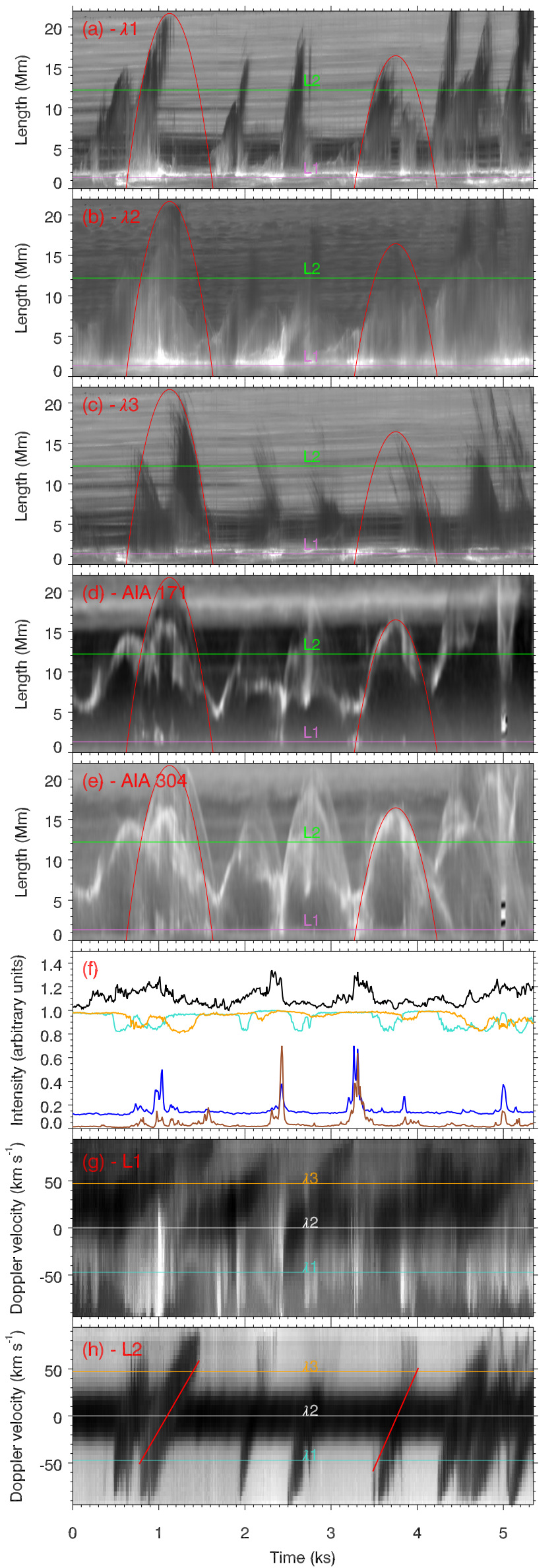

Fig. 5. Same as in Fig. 4 for the yellow trajectory in Fig. 1.
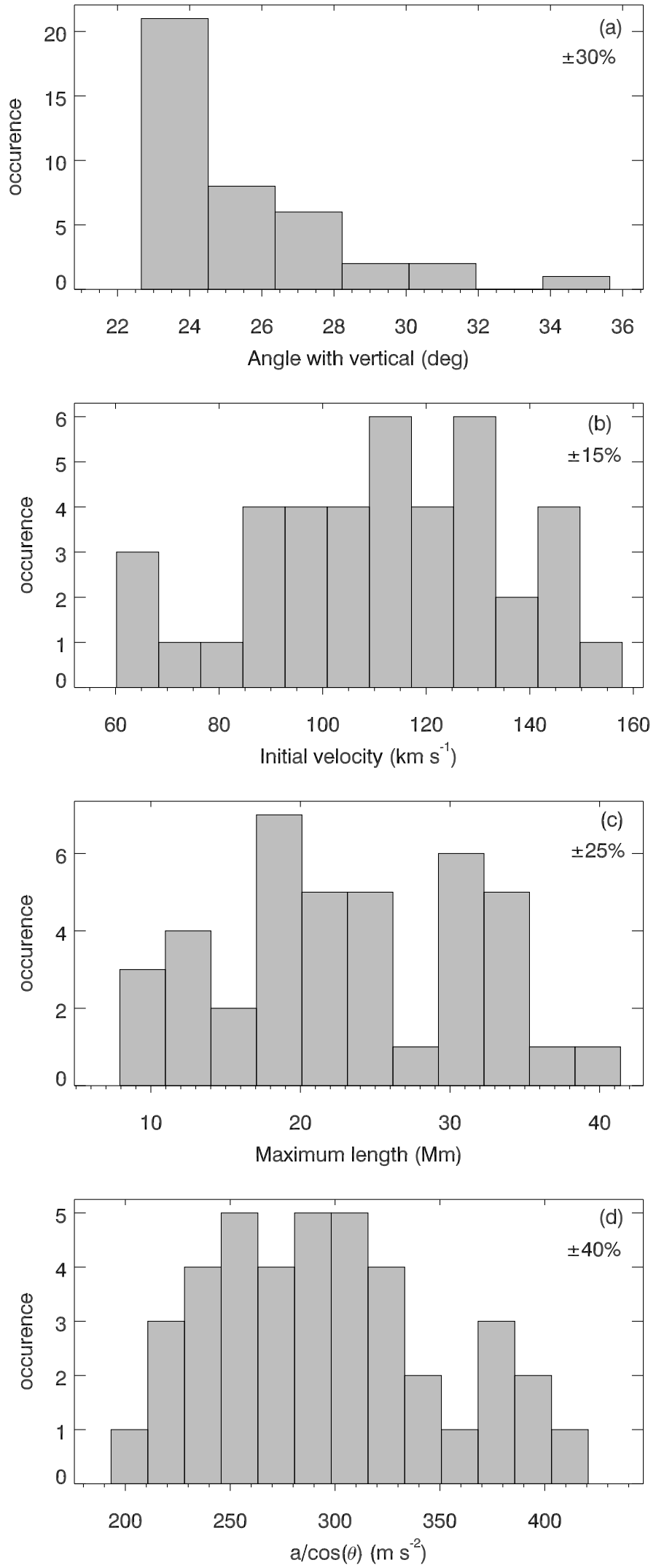

Fig. 6. Histogram of jet properties. The sample size is $N=40$. Relative errors are given in the upper right corner of the panels. a) Angle $\theta$ with the solar vertical; b) inferred initial jet velocity; c) maximum jet extent; d) $a / \cos \theta$, the post-launch deceleration divided by the cosine of the angle with the solar vertical. 
the quantities shown in Fig. 6 ranging from $15 \%$ in $v_{0}$ to $40 \%$ in $a / \cos \theta$. The initial velocities are typically ten times the local sound speed or higher. The lengths are consistent with those reported by Roy (1973a) and Asai et al. (2001). The decelerations are consistent with solar effective gravity without significant other forces.

Finally, we investigated the thermal properties of the acceleration sites. The jet footpoints are not bright in the HMI continuum (see Fig. 2), suggesting no or weak heating in the deep photosphere, but appear bright in AIA $1700 \AA$. We computed the brightness temperature $T_{\mathrm{B}}$ of the $\mathrm{H} \alpha$ data using calibration of a quiet region in the field of view (FOV) with an atlas profile (Neckel \& Labs 1984) and correcting for solar limb darkening (Neckel \& Labs 1994).

Figure 7 shows example profiles in the LB. Dark places typically show brightness temperatures of $5.5 \mathrm{kK}$ in the wings and a normal unshifted line core. The line profiles in the jet footpoints are very different. They typically have bright wings $\left(T_{\mathrm{B}} \sim 5.5 \mathrm{kK}-7 \mathrm{kK}\right)$ and a wide core, somewhat reminiscent of "moustache profiles" in Ellerman bombs (Ellerman 1917). The line core absorption is caused by overlying jet material (Fig. 2). The strongest brightenings have a $T_{\mathrm{B}}$ up to $8 \mathrm{kK}$, and show weak redshifted line-cores and maximum emission on the blue side of nominal line center. Assuming that the brightness temperature is a fraction of the gas temperature, which is reasonable given a source function with a scattering and a thermal component, then the foot-point material visible in $\mathrm{H} \alpha$ is hotter than $8 \mathrm{kK}$. The strongest $\mathrm{H} \alpha$ brightenings are also visible in the AIA channels, typically as the footpoint of an elongated feature (such as the point marked " 1 " in Fig. 2). We therefore used the AIA channels to compute the differential emission measure (DEM) using the method described in Hannah \& Kontar $(2012)^{1}$. The limited number of AIA channels and their wide temperature response limits the accuracy of the DEM analysis. An additional complication is that the He II $304 \AA$ line dominating the AIA 304 channel does not form under coronal equilibrium conditions (see, e.g., Golding et al. 2014), limiting the reliability at temperatures below $100 \mathrm{kK}$. Nevertheless, we show the results for the bright point labeled " 1 " in Fig. 2 in the bottom panel of Fig. 7 and compare it with a typical DEM distribution for the inside of a jet ("2"), the jet front ("3"), and the average over an area in the lower left corner of the SST FOV. The footpoint DEM is two orders of magnitude higher at a temperature of $100 \mathrm{kK}$. This is suggestive of transition region temperatures down at the acceleration site in extreme cases. The AIA brightening at the jet front has a higher than typical DEM, which supports the idea of compression of coronal material.

\section{Discussion and conclusions}

We investigated recurrent jets (which we dubbed peacock jets) of cool $(<15 \mathrm{kK})$ material that are launched along the light bridge of a sunspot in AR11785. Using imaging spectroscopy we measured both the LOS and POS components of the velocity and deceleration of the jets. The POS components are impulsively accelerated in the upper photosphere and/or chromosphere above the sunspot to speeds of up to $145 \mathrm{~km} \mathrm{~s}^{-1}$, decelerate at solar effective gravity, and reach lengths of up to $40 \mathrm{Mm}$. The jets have an angle of about $25^{\circ}$ with the solar vertical. The ballistic motion of the peacock jets is not in agreement with the results of

\footnotetext{
1 Code available at http://www.astro.gla.ac.uk/ iain/ demreg/
}
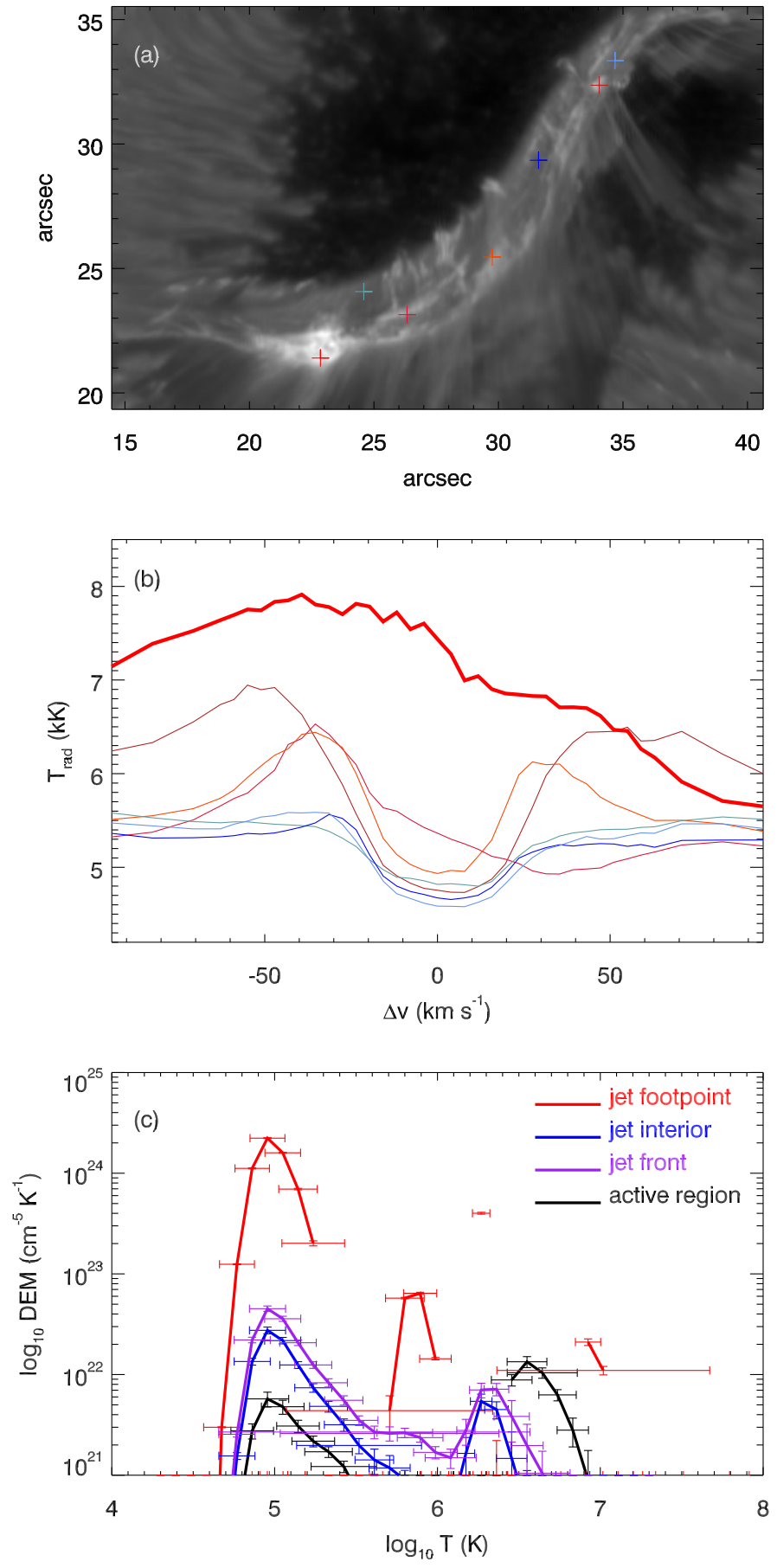

Fig. 7. Top: $\mathrm{H} \alpha$ image of the LB at $\Delta v=-50 \mathrm{~km} \mathrm{~s}^{-1}$; locations of the line profiles in the middle panel are indicated by plus-symbols. Middle: typical $\mathrm{H} \alpha$ profiles in the dark part of the light bridge (shades of blue), and typical profiles in the bright jet footpoints (shades of red). The thick red curve is the profile of the pixel labeled 1 in Fig. 2. Bottom: DEM distribution for the pixels labeled 1-3 in Fig. 2 and an average active region area.

Roy (1973a), who observed extended acceleration followed by a braking stronger than gravity and eventually a fall toward the solar surface with an acceleration smaller than gravity. The reason for this discrepancy lies in a different approach: (1) we do not need to assume a potential field to retrieve the LOS velocity component; (2) we have a sample that is ten times larger; (3) our data have a higher resolution and higher cadence; and (4) our 
deceleration uncertainty is obtained from a proper error analysis. We believe that the results obtained by Roy (1973a) are affected by small number statistics and an overly optimistic error estimate, and we note that the curves in his Fig. 2 could reasonably well be fitted with a parabola.

The accuracy of our derived velocity and deceleration is mainly set by the accuracy with which the Doppler shift of the jet material can be determined. The POS component of the motion can be derived with smaller errors. It would therefore be of value to observe peacock jets close to the limb where the jet axes are more aligned with the POS in order to derive more accurate values of the deceleration and so check whether gas pressure gradients or other forces significantly lower the deceleration as it does in shock-driven dynamic fibrils in plage (De Pontieu et al. 2007) and the chromosphere of sunspots (Rouppe van der Voort \& de la Cruz Rodríguez 2013).

The jet fronts appear bright in the AIA 304, 211, 193, 171, and $131 \AA$ channels, and intermittently even in the $94 \AA$ channel. Supported by our DEM modeling we tentatively interpret this as the effect of shock compression of transition region and coronal material as the cool jet slams into the corona. This is in contrast with Asai et al. (2001) who interpret the bright rim seen in $171 \AA$ around the $\mathrm{H} \alpha$ jets as evidence of a magnetic loop. We find this scenario unlikely as it would require the $\mathrm{H} \alpha$ jets to move at right angles with the magnetic field.

The most interesting aspect of the jets is their acceleration mechanism. Jets are launched with speeds in the range $70-145 \mathrm{~km} \mathrm{~s}^{-1}$, which is nearly 10 times the sound speed at a temperature of $10 \mathrm{kK}$. These high speeds are hard to reconcile with magneto-acoustic shock driving. Shock driven events such as dynamic fibrils exhibit much lower velocities (up to $35 \mathrm{~km} \mathrm{~s}^{-1}$; see Hansteen et al. 2006; De Pontieu et al. 2007; Rouppe van der Voort \& de la Cruz Rodríguez 2013). In contrast, speeds on the order of $100 \mathrm{~km} \mathrm{~s}^{-1}$ are found in smallscale penumbral jets (Katsukawa et al. 2007), which are driven by reconnection between the horizontal dark penumbral filament field and the more vertical bright penumbral field. The peacock jets are mainly launched from the edge of the light bridge where the magnetic field changes rapidly from vertical to horizontal. Magnetic reconnection thus appears to be the only viable candidate mechanism. The following facts also support the reconnection scenario: the acceleration site heating is impulsive, as shown by the sudden increase in $\mathrm{H} \alpha$ brightness at the footpoints, and the jets are predominantly launched from only one side of the LB. This is consistent with the asymmetry induced by the slightly inclined umbral field; we observe jet material flowing down to the other edge of the LB, in agreement with models where cool jet material can slide down along post-reconnection field lines (Yokoyama \& Shibata 1995, 1996; Moreno-Insertis et al. 2008).

We do not believe that peacock jets are caused by the submergence of the umbral field by LB convection, a scenario proposed by Lagg et al. (2014) for granular LBs, as this process would lead to jet acceleration at both LB edges, which is not observed. We are convinced that the data we analyzed show essentially the chromospheric and coronal aspects of the phenomenon observed by Shimizu et al. (2009) and Louis et al. (2014). These authors observe short jets above sunspot light bridges using Hinode SOT Ca II H and study the corresponding photospheric magnetic field using observation with Hinode SP. Shimizu et al. (2009) speculate that the LB could harbor a twisted horizontal flux tube embedded below the canopy formed by the strong umbral field. However, given the constraints of the resolution and the atmospheric model, the inclination map in Fig. 2c does not show any evidence of a twisted flux tube.

The observed LB and its field configuration are roughly reproduced in the simulations of Toriumi et al. (2015b). In their simulation it is the result of a convective upflow carrying a horizontal field to the surface. The horizontal field is aligned along the LB and at the boundaries the shear with the pre-existing vertical field can give rise to magnetic reconnection. Our peacock jet observations are consistent with this scenario.

We note that the LB exhibits a filamentary structure (see Fig. 1) somewhat similar to penumbral filaments and penumbral intrusions. The chromosphere above the latter two can also show jets (Katsukawa et al. 2007; Bharti et al. 2015).

The appearance of the acceleration sites in $\mathrm{H} \alpha$ and the SDO channels is remarkably similar to that of Ellerman bombs (Vissers et al. 2013; Rutten et al. 2013), so lessons learned from Ellerman bombs might be applicable to the acceleration of peacock jets. Coordinated SST and IRIS spectroscopy indicate that Ellerman bombs are sites where the photospheric gas underneath the chromospheric canopy is heated sufficiently to momentarily reach stages of ionization normally assigned to the transition region and the corona (Vissers et al. 2015). We speculate that equally vigorous reconnection might happen at the peacock jet footpoints, leaving a similar imprint in the line profiles. The acceleration sites also share similarities with the hot explosions described in Peter et al. (2014): magnetic reconnection taking place in the upper photosphere heats the surrounding plasma to temperatures normally associated with the transition region (but see Judge 2015) with cold plasma on top. Ellerman bombs and the events reported in Peter et al. (2014) are not associated with clear high-reaching upward moving jets, in contrast to peacock jets. Most likely the vertical magnetic field above the umbra allows for efficient jet formation, while the horizontal magnetic canopy above Ellerman bombs and hot pockets inhibits jet formation.

Acknowledgements. The Swedish 1m Solar Telescope is operated by the Institute for Solar Physics of Stockholm University in the Spanish Observatorio del Roque de los Muchachos of the Instituto de Astrofísica de Canarias. Hinode is a Japanese mission developed by ISAS/JAXA, with the NAOJ as domestic partner and NASA and STFC (UK) as international partners. It is operated in cooperation with ESA and NSC (Norway). This research has benefited from discussions at the International Space Science Institute (ISSI). We thank Jayant Joshi for his valuable suggestions. Jaime de la Cruz Rodríguez acknowledges support from the Swedish Research Council and the Swedish National Space Board.

\section{References}

Asai, A., Ishii, T. T., \& Kurokawa, H. 2001, ApJ, 555, L65

Berger, T. E., \& Berdyugina, S. V. 2003, ApJ, 589, L117

Bharti, L. 2015, MNRAS, 452, L16

Bharti, L., Rimmele, T., Jain, R., Jaaffrey, S. N. A., \& Smartt, R. N. 2007, MNRAS, 376, 1291

Bharti, L., Solanki, S. K., \& Hirzberger, J. 2015, ApJ, submitted [arXiv: 1509.02123]

de la Cruz Rodríguez, J., Löfdahl, M. G., Sütterlin, P., Hillberg, T., \& Rouppe van der Voort, L. 2015, A\&A, 573, A40

De Pontieu, B., Hansteen, V. H., Rouppe van der Voort, L., van Noort, M., \& Carlsson, M. 2007, ApJ, 655, 624

Ellerman, F. 1917, ApJ, 46, 298

Fossum, A., \& Carlsson, M. 2005, ApJ, 625, 556

Golding, T. P., Carlsson, M., \& Leenaarts, J. 2014, ApJ, 784, 30

Hannah, I. G., \& Kontar, E. P. 2012, A\&A, 539, A146

Hansteen, V. H., De Pontieu, B., Rouppe van der Voort, L., van Noort, M., \& Carlsson, M. 2006, ApJ, 647, L73

Judge, P. G. 2015, ApJ, 808, 116

Katsukawa, Y., Berger, T. E., Ichimoto, K., et al. 2007, Science, 318, 1594

Kosugi, T., Matsuzaki, K., Sakao, T., et al. 2007, Sol. Phys., 243, 3 
C. Robustini et al.: Fan-shaped jets above the light bridge of a sunspot driven by reconnection

Lagg, A., Solanki, S. K., van Noort, M., \& Danilovic, S. 2014, A\&A, 568, A60

Lemen, J. R., Title, A. M., Akin, D. J., et al. 2012, Sol. Phys., 275, 17

Louis, R. E., Bayanna, A. R., Mathew, S. K., \& Venkatakrishnan, P. 2008, Sol. Phys., 252, 43

Louis, R. E., Bellot Rubio, L. R., Mathew, S. K., \& Venkatakrishnan, P. 2009, ApJ, 704, L29

Louis, R. E., Beck, C., \& Ichimoto, K. 2014, A\&A, 567, A96

Moreno-Insertis, F., Galsgaard, K., \& Ugarte-Urra, I. 2008, ApJ, 673, L211

Neckel, H., \& Labs, D. 1984, Sol. Phys., 90, 205

Neckel, H., \& Labs, D. 1994, Sol. Phys., 153, 91

Peter, H., Tian, H., Curdt, W., et al. 2014, Science, 346, 1255726

Rouppe van der Voort, L., \& de la Cruz Rodríguez, J. 2013, ApJ, 776, 56

Roy, J.-R. 1973a, Sol. Phys., 32, 139

Roy, J.-R. 1973b, Sol. Phys., 28, 95

Rutten, R. J., Vissers, G. J. M., Rouppe van der Voort, L. H. M., Sütterlin, P., \& Vitas, N. 2013, J. Phys. Conf. Ser., 440, 012007

Sakurai, T., Makita, M., \& Shibasaki, K. 1985, in Theoretical Problems in High Resolution Solar Physics, ed. H. U. Schmidt, 313
Scharmer, G. B., Bjelksjo, K., Korhonen, T. K., Lindberg, B., \& Petterson, B. 2003, in Proc. SPIE, 4853, 341

Scharmer, G. B., Narayan, G., Hillberg, T., et al. 2008, ApJ, 689, L69

Scherrer, P. H., Schou, J., Bush, R. I., et al. 2012, Sol. Phys., 275, 207

Shimizu, T., Katsukawa, Y., Kubo, M., et al. 2009, ApJ, 696, L66

Skumanich, A., \& Lites, B. W. 1987, ApJ, 322, 473

Title, A. M., Frank, Z. A., Shine, R. A., et al. 1993, ApJ, 403, 780

Toriumi, S., Katsukawa, Y., \& Cheung, M. C. M. 2015a, ApJ, 811, 137

Toriumi, S., Cheung, M. C. M., \& Katsukawa, Y. 2015b, ApJ, 811, 138

Tsuneta, S., Ichimoto, K., Katsukawa, Y., et al. 2008, Sol. Phys., 249, 167

van Noort, M., Rouppe van der Voort, L., \& Löfdahl, M. G. 2005, Sol. Phys., 228, 191

Vissers, G., \& Rouppe van der Voort, L. 2012, ApJ, 750, 22

Vissers, G. J. M., Rouppe van der Voort, L. H. M., \& Rutten, R. J. 2013, ApJ, 774, 32

Vissers, G. J. M., Rouppe van der Voort, L. H. M., Rutten, R. J., Carlsson, M., \& De Pontieu, B. 2015, ApJ, 812, 11

Yokoyama, T., \& Shibata, K. 1995, Nature, 375, 42

Yokoyama, T., \& Shibata, K. 1996, Astrophys. Lett. Comm., 34, 133 